\title{
COMPARATIVE EVALUATION OF MICROLEAKAGE AND SHEAR BOND STRENGTH OF BIOACTIVE DENTIN SUBSTITUTE AND NANOHYBRID COMPOSITE RESIN
}

\author{
Ola Barakat* and Muhammad A Samman**
}

\begin{abstract}
Background: In clinical practice, the restoration of non-carious cervical lesions is a challenge, because most of the time the cervical margin is located in cementum or dentin. This characteristic makes the cervical margin more susceptible to microleakage, causing marginal stains, postoperative sensitivity and recurrent caries.

Aim: To compare the microleakage and bond strength for teeth restored with Activa Bioactive restorative and nanohybrid composite resin

Materials and methods: Twenty permanent premolars were selected with class V cavities for microleakage, and 20 permanent premolars were selected for shear bond strength. Microleakage was checked using dye penetration method under digital microscope Universal testing device was used to assess the shear bond strength. Leakage scores, mean and standard deviation values for shear bond strength were calculated from the recorded values. For numerical data student t-test was performed to detect the significance between both groups. For categorical data analysis Chi square test was done.
\end{abstract}

Results; There was non-significant difference between ACTIVA bioactive restorative group and nanohybrid composite resin group in both parameters; leakage and bond strength

Conclusion: Based on the results of the present study, the type of restorative material did not appear to have a significant influence on microleakage or bond strength

KEYWORD: Active Bioactive, Non-Curious Cervical Lesions, Microleakage

\section{INTRODUCTION}

Traditionally, the main objectives for operative dentistry were the eradication of caries, removal of diseased hard and soft tissues, and subsequent restoration with an appropriate material to simulate the anatomy, function, and esthetics of a natural tooth. ${ }^{(1)}$

\footnotetext{
* Associate Professor, Department of Operative Dentistry, Faculty of Oral and Dental Surgery, Misr University For Science and Technology. Egypt

** Associate Professor Dental Biomaterials Department, Faculty of Dental Medicine, Al Azher University, Cairo, Egypt.
} 
Non-carious cervical lesions (NCCLs) are the one of the dominant issues in dentistry, especially among clinical practitioners because of their increasing prevalence. ${ }^{(2)}$ Restoring such lesions is technique sensitive and may lead to failure of cervical adhesive restorations due to a variety of factors, like poor isolation, cuspal deflection during function or bonding to different dental substrates. ${ }^{(3)}$

Variety materials are available for restoring non-carious cervical lesions, like Glass ionomer cement, resin modified GIC and composite resin. Out of these, the most popular restorative material is composite resin due to its optical and strength properties. However, it has a drawback in the form of polymerization shrinkage, which is the main reason for marginal gap formations and subsequent marginal leakage. ${ }^{(2)}$

There is a constant ongoing research toward the development of an ideal restorative material as well as new adhesives to reduce the microleakage and enhance the bond strength. Among this new class of materials is ACTIVA, a bioactive restorative. The manufacturer claims that ACTIVA is among the first permanent dental restoratives to integrate bioactivity by responding to changes in the oral environment. ${ }^{(4)}$

This bioactive material includes glass particles and a hydrophilic ionic resin matrix that "facilitates the diffusion of calcium, phosphate, and fluoride ions," which in turn react to oral $\mathrm{pH}$ changes. ${ }^{(5)}$ According to the manufacturer, these reactions result in improved mechanical properties and the consequential benefits of enhanced esthetics, durability, antimicrobial qualities, and the creation of chemical bonds for decreased leakage of marginal contaminants. ${ }^{(6)}$

However, despite the advances in these materials, the marginal integrity, which is the most important feature of adhesive restorative materials, remains a challenge. Therefore, the purpose of this in vitro study was to compare the Activa bioactive restorative with the commonly used nanohybrid composite regarding the microleakage and bond shear strength testing. The null hypothesis was that, there is no difference in the performance of the two materials

\section{MATERIALS AND METHODS}

Forty freshly extracted human premolar teeth were used in this study. Twenty teeth were selected for microleakage test and the remaining 20 teeth were used for shear bond strength test

\section{Microleakage}

Twenty freshly extracted human premolar teeth were collected from the outpatient clinics of Oral and Maxillofacial Surgery Department, Faculty of Dental Medicine, Alazhar University. Any extrinsic stains or calculus deposits on the teeth were cleaned and specimens were stored in a $1 \%$ chloramine-T solution consisting of $12 \%$ active chlorine diluted in tap water at room temperature. All teeth were examined macroscopically and microscopically ( $\times 20$ magnification) to rule out the presence of fractures, fissures, carious lesions, abrasive or erosive lesions, and restorations.

\section{Cavity design}

Standardized Class V cavities were prepared on the facial or lingual surface at the cementoenamel junction; occlusal margins were located in enamel and apical margins were in cementum. The preparations were cut with a No. 56 carbide bur in a high-speed handpiece cooled with an air-water spray. A No. 257 diamond bur was used to place a 45-degree, 0.5-mm-wide bevel on the enamel margin. Each carbide bur was discarded following preparation of each group of teeth. Preparation dimensions; $2 \mathrm{~mm} \times 3 \mathrm{~mm} \times 2 \mathrm{~mm}$ ( $2 \mathrm{~mm}$ occlusogingivally, $3 \mathrm{~mm}$ mesio-distally $\& 2 \mathrm{~mm}$ in depth) The dimensions of the prepared cavities were standardized using Vernier caliper (accuracy \pm $0.01 \mathrm{~mm})$. 


\section{Restorative procedures and study groups}

The 2 experimental groups were based on the 2 restorative materials: ACTIVA bioactive restorative and nanohybrid composite resin, A2 shade. Each material was used in accordance with the manufacturer's instructions. Both restorative materials were polymerized with a LED curing light (Bluephase G2, Ivoclar Vivadent). The light had been previously monitored with a radiometer and displayed adequate intensity levels $(800 \mathrm{~mW} / \mathrm{cm})$.

\section{Group 1}

Activa bioactive restorative tooth surfaces (enamel and dentin) were conditioned for 10 seconds using Etch-Rite 38\% phosphoric acid etching gel (Pulpdent). The surfaces were rinsed and dried with compressed. air, removing all excess moisture without desiccating the dentin. structure. ACTIVA restorative was dispensed into the preparation in bulk increment and light polymerized for 20 seconds.

\section{Group 2}

Nanohybrid composite resin tooth surfaces (enamel and dentin) were conditioned using 37\% tooth conditioner gel (Dentsply Sirona). The surfaces were rinsed and dried with compressed air, removing all excess. moisture without desiccating the dentin structure. Tetris N Bond (Ivoclar Vivadent, Colombia) was applied to the dentin surface, a wet surface was maintained for 20 seconds, and then the dentin was gently dried with compressed air for 5 seconds. The surfaces were then light. polymerized for 10 seconds. Nanohybrid composite resin restorative was applied in bulk increment and light cured for 20 seconds.

The restorations were polished with SofLex flexible aluminum oxide discs of decreasing abrasiveness from coarse to superfine (3M ESPE) after which the specimens were stored in $100 \%$ humidity for 24 hours at room temperature prior to leakage assessment.

\section{Thermo-mechanical aging and dye immersion}

The samples were subjected to thermocycling in a water bath for 500 cycles between $5^{\circ}$ and $55^{\circ} \mathrm{C}$ with a dwell time of $25 \mathrm{~s}$ in each water bath (Robota automated thermo-cycle; BILGE, Turkey) followed by subjecting them to cyclic loading with 196 Newton force for 10,000 cycles at frequency $1 \mathrm{~Hz}$ using universal testing device (Instron 3345, USA) (2) Following this, all the surfaces of the samples were double coated with nail varnish, except a1 mm margin around the restoration and their apices were sealed with wax. All specimens were immersed in $2 \%$ methylene blue dye solution for $24 \mathrm{hrs}$ after which they were rinsed with water and air-dried. The specimens were invested in auto polymerizing resin (Acrostone, Cairo, Egypt) and coded.

\section{Microleakage assessment}

The specimens were longitudinally sectioned in a buccolingual direction using a low-speed (1600 rpm) linear precision saw (Isomet 5000, Precision saw, Buehler USA) with a diamond-coated blade. The sectioned restorations were examined under USB digital-microscope (U500x Digital Microscope, Guangdong, China) at 30Xmagnification. The depth of dye penetration was analyzed based on a modified scoring system. ${ }^{(7)}$

Score 0 , no leakage; score 1, leakage up to onehalf the length of the cavity wall; score 2 , leakage along the full length of the cavity wall, not including the axial surface; or score 3, leakage along the full length of the cavity wall, including the axial surface (Figure2).

\section{Shear Bond Strength}

Twenty permanent premolars were divided into two groups and horizontal indentations were made on radicular portion. The auto polymerizing acrylic resin blocks were made by Teflon molds with the root portion of each tooth embedded and crown portion exposed and parallel to the base. A low- 
speed Isomet fine diamond disk with copious water spray was used to expose superficial dentin of the occlusal surface, cut perpendicular to the long axis of the tooth. Then 400 grit aluminum oxide (Automata Grinding, GmbH, Germany) abrasive paper was used to obtain flat dentin surface. Specimens were then stored in distilled water at room temperature.

All specimens were rinsed with distilled water and air dried directly before the application of the test material using the polyethylene tube (4-mm diameter, 2-mm height) as mentioned in leakage test. Care was taken to avoid any air entrapments, voids, or gaps. After completion of curing, the polyethylene tubes were removed with a sharp knife. All specimens were incubated at $37^{\circ} \mathrm{C}$ in water for $24 \mathrm{~h}$. The samples were subjected to thermocycling as previously mentioned in leakage test.

\section{Measurement of shear bond strength}

A circular interface shear test was designed to evaluate the bond strength. All samples were individually and horizontally mounted on a computer-controlled material testing machine (Model 3345; Instron Industrial Products Norwood, USA) with a loadcell of $5 \mathrm{kN}$ and data were recorded using computer software (Bluegill Lite; Instron Instruments).

Samples were secured to the lower fixed compartment of testing machine by tightening screws through metallic custom-made housing device with central cavity into which the acrylic block fit (dimensions;25x25 mm). Shearing test was done by compressive mode of load applied at resintooth interface using a mono-beveled chisel metallic rod attached to the upper movable compartment of testing machine traveling at crosshead speed of 0.5 $\mathrm{mm} / \mathrm{min}$ (Figure 1). The load required to de-bonding was recorded in Newtons.

Shear bond strength calculation; The load at failure was divided by bonding area to express the

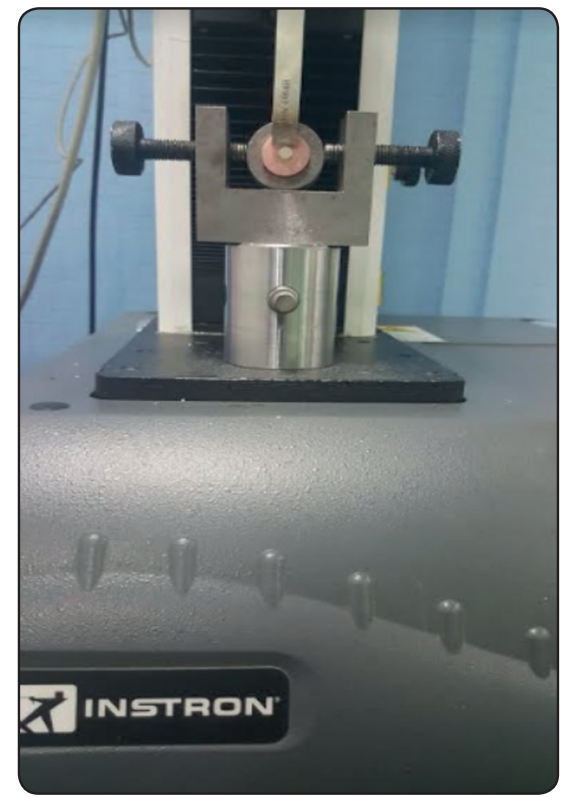

Fig. (1) Showing shear bond strength sample mounted onto testing machine

bond strength in $\mathrm{MPa} ; \tau=\mathrm{P} / \pi \mathrm{r} 2$ where $; \tau=$ shear bond strength $(\mathrm{MPa}), \mathrm{P}=$ load at failure $(\mathrm{N}), \pi=3.14$ and $\mathrm{r}=$ radius of composite $\operatorname{disc}(\mathrm{mm})$

Failure analysis; The de-bonded test specimens were examined under USB digital-microscope at a magnification of $\times 25$ and fractures were classified as follows: Cohesive failure - failure within restoration or dentin, adhesive failure - failure at restorationdentin interface, and mixed failure - When two modes of failure occur simultaneously.

Statistical analysis; statistical analysis was performed by using MS Excel 2010 and Graph Pad Instat (Graph Pad, Inc.) software for windows. A value of $\mathrm{P}<0.05$ was considered statistically significant. For numerical data student t-test was performed to detect the significance between both groups. For categorical data analysis Chi square test was done. Sample size $(n=10)$ was large enough to detect large effect sizes for main effects and pairwise comparisons, with the satisfactory level of power set at $80 \%$ and a $95 \%$ confidence level. 


\section{RESULTS}

\section{Microleakage}

Table (1) summarized the distribution of microleakage scores for both groups. Representative microphotographs of Class $\mathrm{V}$ restorations (magnification $\times 30$ ) showing different microleakage scores in figure (2).

For Activa bioactive composite group, score 0 recorded the highest \% (40\%) followed by score $1(30 \%)$ then score $2(20 \%)$ while the lowest $\%$ recorded for score 3 (10\%). For Nanohybrid composite group, score 0 recorded the highest \% (50\%) followed by score $1(30 \%)$ while the lowest $\%$ recorded for scores 2 and 3 (10\% for each). The difference between both groups was statistically non-significant $(\mathrm{p}=0.8327>0.05)$ as confirmed by Chi square test, table (1)

\section{Shear bond strength}

The mean shear bond strength and standard deviation are shown in Table (2) and graphical drawn in figure (3 ).It was found that Nanohybrid composite group recorded statistically significant higher shear bond strength mean value $(15.449 \pm 1.88$ $\mathrm{MPa})$ than Activa bioactive composite group $(9.447 \pm 1.05 \mathrm{MPa})$ as indicated by unpaired t-test $(\mathrm{P}=0.05)$.

The observed modes of failure were predominantly adhesive or mixed failure in Activa bioactive composite group and little cohesive failure. Nanohybrid composite group showed majority of mixed and adhesive failures while minority record for cohesive one. Chi square test showed nonsignificant difference in failure mode distribution between groups $(\mathrm{p}=0.3292<0.5)$.

TABLE (1) Comparison between frequent distribution of Leakage score results recorded for both groups

\begin{tabular}{|c|c|c|c|c|c|c|}
\hline \multirow{2}{*}{\multicolumn{2}{|c|}{ Variable }} & \multicolumn{4}{|c|}{ Microleakage scores } & \multirow{2}{*}{$\frac{\text { Statistics }}{\text { P value }}$} \\
\hline & & Score 0 & Score 1 & Score 2 & Score 3 & \\
\hline \multirow{2}{*}{ Composite resin } & Bioactive & $4(40 \%)$ & $3(30 \%)$ & $2(20 \%)$ & $1(10 \%)$ & \multirow{2}{*}{$0.8327 \mathrm{~ns}$} \\
\hline & Nanohybrid & $5(50 \%)$ & $3(30 \%)$ & $1(10 \%)$ & $1(10 \%)$ & \\
\hline
\end{tabular}

ns; non-significant ( $p>0.05)$

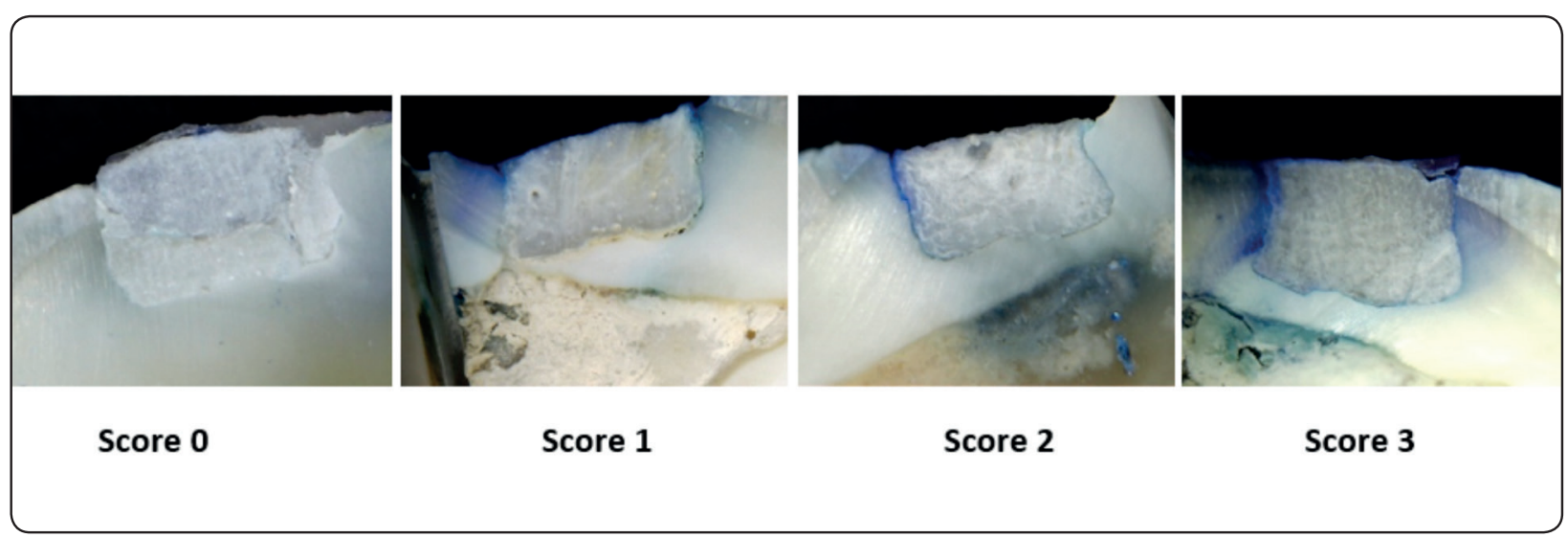

Figure (2) Representative microphotographs of Class V showing different microleakage scores 
TABLE (2) Comparison of shear bond strength results (Mean \pm SD) and failure analysis (\%) between groups

\begin{tabular}{|c|c|c|c|c|c|}
\hline \multicolumn{2}{|c|}{ Variables } & Shear bond strength & \multicolumn{3}{c|}{ Failure mode } \\
\cline { 3 - 6 } & Mean \pm SD & Adhesive & Cohesive & Mixed \\
\hline \multirow{2}{*}{$\begin{array}{c}\text { Composite } \\
\text { resin }\end{array}$} & Bioactive & $9.447 \pm 2.173$ & $5(50 \%)$ & $1(10 \%)$ & $4(40 \%)$ \\
\cline { 2 - 6 } & Nanohybrid & $11.896 \pm 2.975$ & $4(40 \%)$ & $1(10 \%)$ & $5(50 \%)$ \\
\hline Statistics & P value & $0.05 \mathrm{~ns}$ & \multicolumn{4}{|c}{$0.3292 \mathrm{~ns}$} & \\
\hline
\end{tabular}

ns; non-significant ( $p>0.05)$

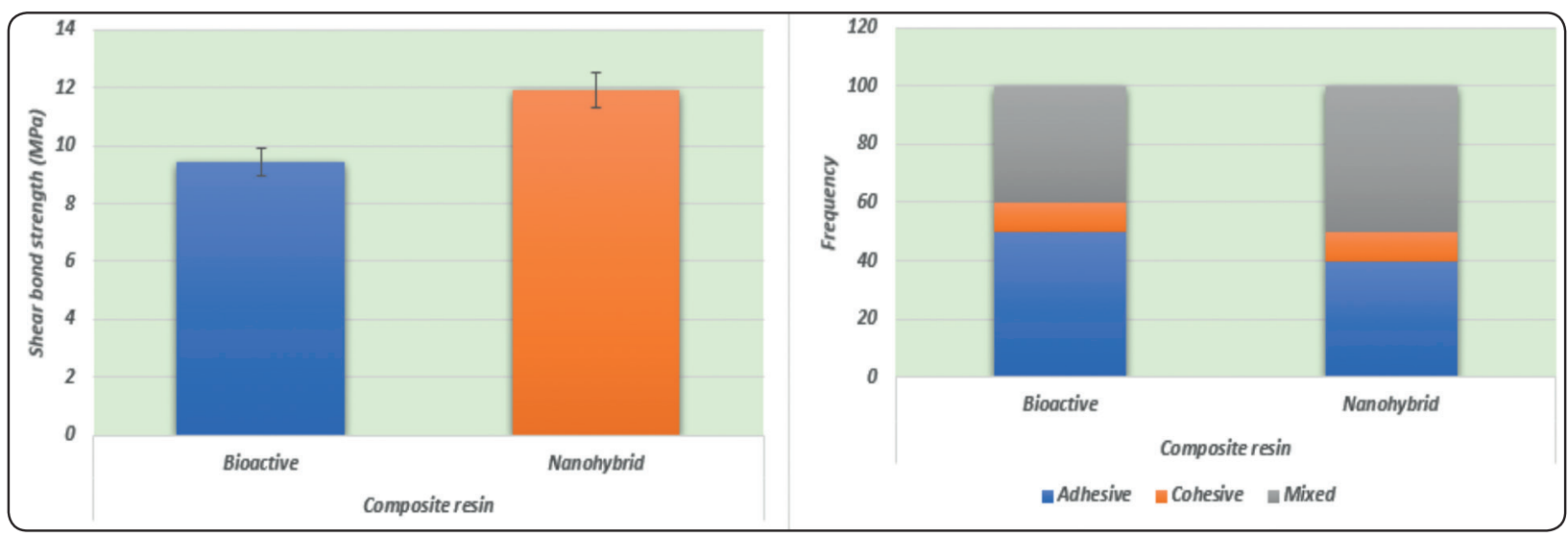

Fig. (3) Showing shear bond strength means values (a) and failure mode distribution (b) for both groups

\section{DISCUSSION}

Dental restoratives-including adhesive materials and composite resins-have developed from a "generationally" informed hierarchy of technological advancement. Marketing solutions have relied heavily on variation within the alteration of the physical properties and completely different material constituents, including packability, flowability, various insertion techniques; incremental vs bulk, and restoration delivery methods; thermal and/or sonic energy. ${ }^{(8,9,10,11)}$

In 2013 Pulpdent ${ }^{\circledR}$ Corporation acquired the premarket approval from the US Food and Drug Administration (FDA) to introduce new bioactive restorative materials in attempt to overcome the disadvantages of GIC and RBC and combine their advantages in one restorative material, namely ACTIVA BioACTIVE ${ }^{\mathrm{TM}}$. Those materials are ionic composite resins which combine the biocompatibility, chemical bond and the ability to release fluoride of GIC with the mechanical properties, aesthetic and durability of RBC..$^{(12,13)}$

Microleakage forms the basis for predicting the performance of any restorative material. Several methods are available for detecting microleakage. These include the use of dyes, chemical tracers, and radioactive tracers, neutron activation analysis, and fluid filtration. A dye leakage method was used in this study as it is simple, reliable and well accepted. ${ }^{(14)}$ Among the various dyes available methylene blue was selected. It has a low molecular, which is smaller than the diameter of a dentinal tubule and has the ability to penetrate through the smallest of gaps between the interface of the tooth and restoration. ${ }^{(15)}$ 
Microleakage, as associated with dental restorations, has been defined as the clinically undetectable passage of bacteria, fluids, molecules, or ions between a cavity wall and the restorative material applied to it. ${ }^{(16)}$

This process can be a consequence of several factors. ${ }^{(17)}$ These factors include, but are not limited to, physicochemical properties of the material, polymerization method, and outline and form of the cavity preparation. In addition, the occurrence of microleakage can be influenced by operator (technique) variables, including material manipulation, insertion procedures, isolation limitations, and observance of the fundamental requirements of dental adhesive and composite resin technology. Eventual sequelae of microleakage include marginal discoloration, micro-gap formation, recurrent caries, possible pulpal involvement, and restoration replacement. ${ }^{(18)}$

Due to fluctuation in temperatures, when the restorative materials are placed in the oral environment, they are constantly exposed to thermal variations which may affect their clinical performance. Thus, to simulate temperature changes, specimens were subjected to thermocycling procedures. ${ }^{(19)}$

The mechanical aging via cyclic loading was performed as the occlusal stresses produced in tooth during normal function and parafunction may increase microleakage and deteriorate the marginal integrity of restorations. ${ }^{(20)}$ Class $\mathrm{V}$ cavities offer a good experiment for testing adaptation of material to the tooth substrate. The cervical lesions pose a restorative challenge due to the complex morphology. ${ }^{(21)}$

There are numerous methods for assessing the adhesion of a dental material to dentin, including tensile, shear, and push-out bond strength tests. In this study shear bond strength test was done based on its relative easiness, simplicity, inexpensive and reproducibility compared to tensile bond testing and to avoid friction effect that occurs in push out bondtesting. ${ }^{(22)}$
The present study was designed to compare between microleakage and shear bond strength of Bioactive Dentin substitute and nanohybrid composite resin.

The results revealed that there was non-significant difference between bioactive restorative composite resin system (ACTIVA) group and nanohybrid composite resin group in both parameters; leakage and bond strength testing.

The results agreed with those reported in previous studies. ${ }^{(15,23)}$ This might be attributed to Activa Restorative contains glass particles and polyacid components of resin-modifed glass ionomer cements, which undergo the acid/base hardening reaction of all glass-ionomer systems. Also, the ionic resin component which contains phosphate acid groups with antimicrobial properties that improve the interaction between the resin and the reactive glass fillers and enhance the interaction with tooth structure. ${ }^{(24)}$ In addition, due to presence of ionic resin matrix, this material category can achieve polymerization by both light cure and chemical cure. The hydrogen ions break off from the phosphate groups through an ionization process that is dependent upon water \& are replaced by calcium in the tooth structure. This ionic interaction binds the resin to the minerals in the tooth, forming a strong resin hydroxyapatite complex and a positive seal. ${ }^{(2)}$ Thus, there are three hardening mechanisms involved with the Activa Restorative. ${ }^{(25)}$

Also, retention achieved with adhesive bonding is augmented by mechanical undercutting as is the case in most cavity preparations. "White line" margins, which are considered typical of freshly finished bonded resin bonded composite restorations, are prominently absent, which is most likely owing to low polymerization shrinkage. ${ }^{(23)}$

Contradictory results in previous study that have employed similar techniques for the same material have often provided. This contrast may have resulted from different handling and manipulation of materials. ${ }^{(2)}$ 


\section{CONCLUSION}

Within the limitations of this in vitro study, the following conclusions might be drawn: the results indicated that use of a bioactive restorative system in Class $\mathrm{V}$ cavity preparations would result in comparable microleakage and interfacial adhesion to a nanohybrid composite resin. Thus, it may be considered as a good restorative option for restoring carious lesions. The result from in vitro studies cannot necessarily be extrapolated to in vivo results.

\section{REFERENCE}

1. Perdigão J. New developments in dental adhesion. Dent Clin North Am. 2007;51(2):333-357.

2. Kaushik M, Yadav M. Marginal Microleakage Properties of Activa Bioactive Restorative and Nanohybrid Composite Resin Using Two Different Adhesives in Non Carious Cervical Lesions - An In Vitro Study. Journal of the west african college of surgeons. 2017; 7(2): 1-14.

3. Gerdolle D, Mortier E, Droz D. Microleakage and Polymerization Shrinkage of Various Polymer Restorative Materials. Journal of Dentistry for Children.2008;75(2):125-133.

4. Sonarkar S, Purba R. Bioactive materials in conservative dentistry. Int J Contemp Dent Med Rev. 2015; 340115.

5. Chen L, Shen H, Suh BI. Bioactive dental restorative materials: a review. Am J Dent. 2013;26(4):219-227.

6. Pulpdent. ACTIVA BioACTIVE [white paper]. 3rd ed June 2017. https://www.pulpdent.com/wp

7. Franco EB, Gonzaga Lopes L, Lia Mondelli RF, da Silva Souza MH Jr, Pereira Laurie JR. Effect of the cavity configuration factor on the marginal microleakage of esthetic restorative materials. Am J Dent.2003;16(3):211-4.

8. Bonilla ED, Stevenson RG, Caputo AA, White SN. Microleakage resistance of minimally invasive Class I flowable composite restorations. Oper Dent. 2012;37(3):290-298.

9. Kachalia PR. Composite resins 2.0: entering a new age of posterior composites. Dent Today. 2013;32(12):78, 80-81.

10. Karaman E, Ozgunaltay G. Polymerization shrinkage of different types of composite resins and microleakage with and without liner in class II cavities. Oper Dent. 2014;39(3):325-331.

11. Kalmowicz J, Phebus JG, Owens BM, Johnson WW, King GT. Microleakage of Class I and II composite resin resto- rations using a sonic-resin placement system. Oper Dent 2015;40(6):653-661.

12. Croll TP, Berg JH, Donly KJ. Dental repair material: a resin-modified glass-ionomer bioactive ionic resin based composite. Compend Contin Educ Dent. 2015;36(1):60-5.

13. ACTIVA BioActive product list XF-VPA3 REV 11/2015 https://www.pulpdent.com/shop/category/activabioactive cement/?productlist=activa\&shopp_category=activa-bioactive-cement. 2015.

14. Alani AH, Toh CG. Detection of microleakage around dental restorations: A review. Oper Dent 1997;22:173-85.

15. Owens BM, Phebus JG, Johnson WW. Evaluation of the marginal integrity of a bioactive restorative material. Gen Dent. 2018;66(3):32-36

16. Costa Pfeifer CS, Braga RR, Cardoso PE. Influence of cavity dimensions, insertion technique and adhesive system on microleakage of Class V restorations. J Am Dent Assoc. 2006;137(2):197-202.

17. Kubo S, Kawasaki A, Hayashi Y. Factors associated with the longevity of resin composite restorations. Dent Mater J. 2011;30(3):374-383.

18. Ilie N, Hickel R. Resin composite restorative materials Aust Dent J. 2011;56(Suppl 1):59-66

19. Pazinatto FB, Campos BB, Costa LC, Atta MT. Effect of the number of thermocycles on microleakage of resin composite restorations. Pesqui Odontol Bras2003;17:337-41.

20. Arisu H.D., Uçtasli M.B., Eligüzeloglu E., Ozcan S., Omürlü $\mathrm{H}$. The effect of occlusal loading on the microleakage of class V restorations. Oper Dent. 2008; 33(2):135-141.

21. Owens BM, Johnson WW. Effect of single step adhesives on the marginal permeability of class $\mathrm{V}$ resin restorations. Oper Dent.2007;32:67-72.

22. Wang M, Kornfeld JA. Measuring shear strength. of soft-tissue adhesives. J Biomed Mater Res Part B 2012:100:618-23.

23. Alkhudhairy FI, Ahmad ZH. Comparison of shear bond strength and microleakage of various bulk-fill bioactive dentin substitutes: an in vitro study. J Contemp Dent Pract. 2016;17(12):997-1002

24. Naorungroj S,Wei HH, Arnold RR, Swift EJJr, Walter R. Antibacterial surface properties of fluoride containing resin-based sealants. J Dent 2010;38(5):387-91.

25. The future of dentistry now in your hands. PULPDENT ${ }^{\circledR}$ publication. Watertown, MA: Pulpdent Corporation; 2014. 